\title{
AUTOMATIC EXTRACTION OF ROAD SURFACE AND CURBSTONE EDGES FROM MOBILE LASER SCANNING DATA
}

\author{
A.Miraliakbari, M. Hahn, S. Sok \\ Department of Geomatics, Computer Science and Mathematics, University of Applied Sciences Stuttgart, Schellingstraße 24, 70174 \\ Stuttgart, Germany, - (alvand.miraliakbari, michael.hahn, sokunthet.sok)@hft-stuttgart.de
}

WG IV/7 and WG V/4

KEY WORDS: Automation, Extraction, Laser scanning, Mapping, Mobile, Surface, Urban

\begin{abstract}
:
We present a procedure for automatic extraction of the road surface from geo-referenced mobile laser scanning data. The basic assumption of the procedure is that the road surface is smooth and limited by curbstones. Two variants of jump detection are investigated for detecting curbstone edges, one based on height differences the other one based on histograms of the height data. Region growing algorithms are proposed which use the irregular laser point cloud. Two- and four-neighbourhood growing strategies utilize the two height criteria for examining the neighborhood. Both height criteria rely on an assumption about the minimum height of a low curbstone. Road boundaries with lower or no jumps will not stop the region growing process. In contrast to this objects on the road can terminate the process. Therefore further processing such as bridging gaps between detected road boundary points and the removal of wrongly detected curbstone edges is necessary. Road boundaries are finally approximated by splines.

Experiments are carried out with a ca. $2 \mathrm{~km}$ network of smalls streets located in the neighbourhood of University of Applied Sciences in Stuttgart. For accuracy assessment of the extracted road surfaces, ground truth measurements are digitized manually from the laser scanner data. For completeness and correctness of the region growing result values between $92 \%$ and $95 \%$ are achieved.
\end{abstract}

\section{INTRODUCTION}

Road extraction from aerial images, airborne laser scanning data, and high-resolution satellite images has a long research tradition. The main purpose of those developments was to generate or update roads maps. However, many details of a road surface are not visible in those data. With mobile laser scanning this limitation is overcome. These systems capture the road corridor and generate huge point clouds that describe a road scene in detail.

Road surface extraction from laser scanning data is an important preparatory step for many tasks related to road maintenance and infrastructure planning. The extraction of a road surface is a first step for road condition mapping which is the background of the developments in this paper.

Mobile laser scanning covers sensors and procedures for point cloud acquisition from moving platforms. By mounting the system on road vehicles and driving the vehicles through the city, the recorded point cloud covers road surfaces, curbstones, sidewalks, traffic signs, facades etc. By mounting the scanner such that scan direction is perpendicular to the driving direction, typically a high point density is achieved in transverse direction on the road. This can favourably be used to separate road surfaces from the sidewalks. Often used criteria are height jumps or slope changes at the vicinity of the region boundary. The curbstone height above ground varies typically from $10 \mathrm{~cm}$ to $20 \mathrm{~cm}$ (Wikipedia, 2014). In practice, however, there are many exceptions as shown for example in Figure 1 side of a road protrude around $3 \mathrm{~cm}$ above the road surface.

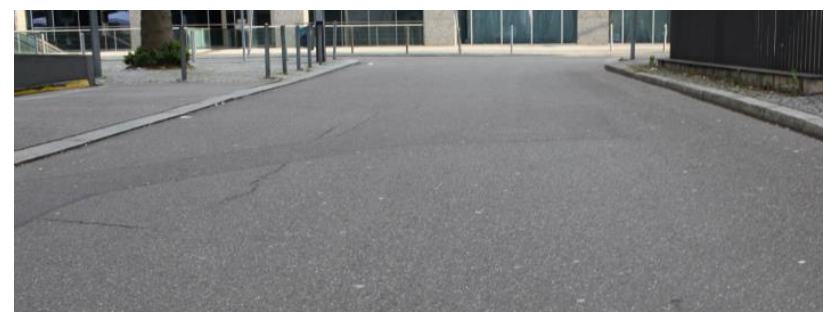

Figure 1. A example of a low curb on the left side of the imaged road

Several related researches have been published over the past decade. (Vosselman and Liang, 2009) investigated the detection of curbstones in airborne laser scanning data. Locations with 'height jumps' are detected which are potential curbstone positions. Moreover they reconstructed the line topology and bridged the gaps in the road boundary outlines. (Samadzadegan et al., 2009) investigated automatic road extraction from airborne Lidar data based on classifier fusion. They employed minimum distance and maximum likelihood classifiers and combined them using weighted majority voting and selective Naïve Bays technique. The authors stated that the Naïve Bays method leads to better outcomes. (Siegemund et al., 2010) investigated curb reconstruction using conditional random field (CRF). The input data they used is a dense 3D point cloud generated from images by stereo matching techniques. These images were taken from the camera mounted in the vehicle windshield. Their suggested workflow operates online. The 3D traffic area with street floor, curbstones and sidewalks is captured. The proposed method is able to reconstruct the curbstones that have different heights from adjacent points on the street. The authors report that they fit straight lines but also higher order polynomials through the detected curbstone edges. (Michalke et al., 2010) examined a self-adaptive method for 
curbstone detection. Their method is activated by processing of 'human neural signal processing'. They fused a multi-sensor which is like human characteristics of observation for online adaptation of road boundaries and curbstones position. Gabor filter kernels are employed for edge (curb line) detection. (Gallo et al., 2008) studied curbstone detection by means of time-offlight (TOF) camera. They employed a camera called Canesta. Their plane-based approach follows a RANSAC version called CC-RANSAC. They stated that contrary to the ordinary RANSAC which considers all inliers, the modified version takes only the largest associated components of inliers into the consideration. They claimed that the proposed approach prevents RANSAC failure if shallow curbs exist in the search region. Also the cost efficiency of their method due to the less required iterations is addressed. (El-Halawany et al., 2011) studied curb line detection using mobile laser scanning data. They first separate ground and non-ground depending on parameters such as direction of the 'surface normal and the size of normalized eigenvalues'. Undesired objects are eliminated, and finally the curb lines are detected based on some 3D segmentation and edge detection techniques.

In the following we pick up ideas on region growing using irregular point clouds and analyse the point cloud locally to develop criteria for the region growing process. The growing results in the street floor region. The region boundaries are mostly but not always curbstone edges which are finally approximated by splines to define the road boundaries.

The concept of our work is outlined in the next section. We present and discuss results in section 3. Summary and an outlook are given in the last section.

\section{CONCEPT}

We consider this study as an initial step for our main goal which is automatic detection of road surface depressions such as ruts, potholes and general unevenness. Readers can find further details in (Miraliakbari et. al., 2014).

In this study, we assume that the road surface has a lower height compared to its environment. So we extract the road surface by detecting height jumps, which we assume to coincide with the street borders (curbstone edges). We examine two variants of jump detection, namely: height difference-based (HDB) and histogram-based (HB) jump detection.

Detected jumps are marked in a symbolic road image. Starting from seed pixels on the road, a region growing is performed for which the detected height jumps serve as stop criteria. However, not in all cases the road border is characterized by a height jump. Junctions of bikeways and streets feature frequently smooth transitions for the convenience of bikers. Such shallow curbs imply difficulties: due to the region growing condition, side walk and bikeways might be detected partially as street floor, because the region growing only stops at facades or other obstacles far outside the road. This causes the detection of false curbstones, while the real road border is missing or interrupted. Similar effects are induced by occlusions of the laser rays by cars etc., which also cause gaps in the curb lines; additionally, in such cases the road is not completely covered by laser points. To overcome these effects, the detected height jumps have to be filtered for outliers and have to be completed to smooth continuous lines. Once the final continuous road borders are found, the road surface is identified as the area between these borders.

The results of the above-mentioned variants for jump detection are compared with each other and with the hand-operated outcomes which we use as ground truth for accuracy assessment.

\subsection{Pre-processing, gross height filtering}

The majority of objects represented by the mobile laser scanning data are non-ground objects which must be removed as much as possible. The scanner trajectory of the dataset is divided in equidistant intervals of 2 meters in order to generate point cloud segments. As the point cloud is denser on the road surface than at other locations, especially close to the foot points of the laser scanner positions on the ground, the most frequent height values are encountered at points located on the street. Figure 2 shows a fraction of the unprocessed point cloud. Pre-processing results are presented in blue. The majority of the points, in each section, are located on the street floor and the pedestrian area. Points located higher than $0.5 \mathrm{~m}$ compared to the location of the highest point density are removed. In Figure 2 , only the points within the blue band are retained.

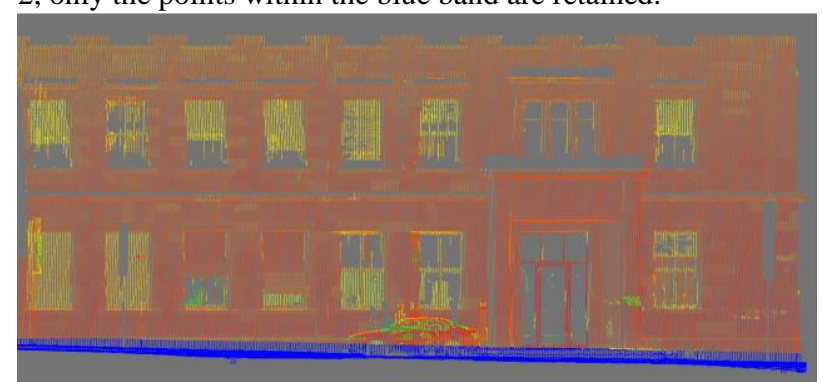

Figure 2. Cross section of a fraction of raw and pre-processed laser scanning data

\subsection{Principle of height jump detection}

In order to find jumps as indications of curbstone edges, we investigate local features of the points. In particular, we surround each point by a vertical cylinder or by cuboid and consider the vertical point distribution within either body. The corresponding histogram represents the frequency per length unit. We introduce two criterion functionals in order to decide whether the neighbourhood contains a jump or not. We call these two variants height difference-based (HDB) and histogram-based (HB) jump detection, respectively.

\subsubsection{HDB}

In this variant, the target function is the difference between maximum minus minimum height of the points located inside the vertical cylinder. Figure 3 shows a schematic representation of the criterion. If the maximal height difference exceeds a certain threshold, the laser points inside the cylinder represent the border between road surface and the sidewalk.

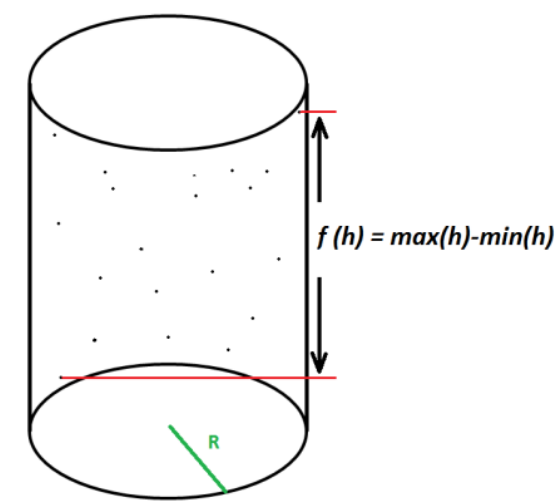

Figure 3. Schematic view of the criterion used in the HDB variant 
In this variant, we have considered only the magnitude of the highest and lowest point along the $\mathrm{z}$-axis without considering the distribution within the cylinder.

\subsubsection{HB}

HB variant replaces the cylinder shown in Figure 3 by equidistant-voxels. The height of the voxels is the same as the predefined threshold. Due to the different longitudinal and transverse intervals of the laser points, the edges of the voxel are not equal. Following algorithm is implemented for this variant;

A) Input the pre-processed point cloud segments. Each segment contains two parts, namely right-scan and leftscan. We call them R-section and L-section.

B) Rotate the scan-lines to be parallel to the Y-axis of the new coordinate system.

C) Calculate the bounding box of each subsection. $\Delta X=\max X-\min X$ and $\Delta Y=\max Y-\min Y$.

D) Voxelize the subsections: Magnitude of edges of voxel elements depends on the regular curbstone height and density of the point cloud. In our research we have specified: $d x_{v}=10 \mathrm{~cm}, d y_{v}=5 \mathrm{~cm}, d z_{v}=2 \mathrm{~cm}$.

E) Associate binary values with the voxel elements. If voxel contains one or more than one laser scanning point, the associated value amounts to one otherwise zero.

F) Sum up the values of each vertical pile above the pixel. The result is a $2 \mathrm{D}$ grid. We classify the grid into three classes; 'zero', 'one' and 'two'. If the result of the summation is more than one, the respective grid cell belongs to class 'two'.

Two constraints must be considered during the region growing process in this variant:

a- If within the region growing, a voxel with value zero is reached, the process will end.

b- In each vertical pile, if there is an empty voxels between nonempty voxels (right above the non-empty voxel), the region growing will be continued. The reason is to avoid confusion of car bumpers with curbstones. Of course one should consider the extra data which remains in the road surface class. The extra data which is in such case part of the car bumpers must be filtered in the post-processing.

Figure 4 shows the schematic presentation of the HB variant. Although as explained in part $(\mathrm{F})$, the summation of the nonempty voxels along this are more than one, but due to having empty voxel in between, the class value amounts to one.

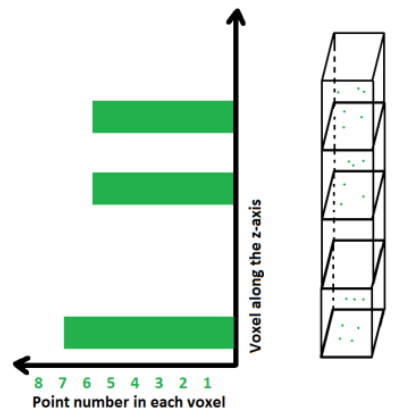

Figure 4. Schematic view of the HB variant

\subsection{Region growing}

We have proposed two region growing variants, namely: fourneighbourhood and two-neighbourhood.

\subsubsection{Four-neighbourhood region growing}

In this region growing, as seed points we employ the grid cells which are closest to the projected scanner trajectory on the ground. Each cell (candidate grid) of the 2D grid overlaid on the 2D coordinates of the point cloud shall be the centre of a circle with a certain radius (used in HDB) or centre of the rectangle (used in $\mathrm{HB}$ ) surrounded by the respective 2D irregular points. If the criterion of the target function discussed in previous subsections is fulfilled, the region growing will be continued; otherwise the candidate grid will be inactivated. For testing the fulfilment of the criterion, $\mathrm{Z}$ coordinate of the point cloud is taken into account.

\subsubsection{Two-neighbourhood region growing}

2.3.3 The major difference of this region growing with the former one is the dimension of region growing. This option offers two-neighbourhood region growing. In fourneighbourhood region growing, if an arbitrary small part of a data segment has shallow curbstone, the four-neighbourhood region growing contaminates the whole sidewalk. Alternatively we shall not obtain such dramatic results in two-neighbourhood variant. In Two-neighbourhood region growing, seed points are again pixels located nearby the $2 \mathrm{D}$ laser scanner positions. The search starts from the lowest row of every column of the Rsection and goes as long as the criterion of either HDB or HB is fulfilled. Same condition applied for L-section.

\subsection{Detection and vectorisation of the curbstone edges}

In order to achieve two aims with one algorithm, we used the result of road surface extraction as an input to detect and vectorise curbstone edges. There are always some gaps in the detected curbstone edges which occur due to the parked cars or nonappearance of curbstones in some special area such as carriage drive or wheelchair locations (Vosselman and Liang, 2009). The gaps must be filled and all positions of the curbstones must be connected to each other.

Results of $\mathrm{HDB}$ and $\mathrm{HB}$ variants are employed for street boundary detection. Using result of the HDB, surrounding laser points of the inactivated candidate cell will be considered as potential curbstone positions. In this variant, the obstacles like car bumpers will wrongly be considered as curbstone edges. In HB variant, after a 'zero' value is encountered (e.g. occlusion), or the grid value is more than 'one' and there is no empty voxel between the pile, the process ends. The grid cell where the region growing comes to halt is considered as potential curbstone edge.

Elimination of the obstacles and refinement of the curbstone edges is the next task. Obstacles, mainly parked vehicles, degrade the process of road boundary detection. Following algorithm shows how to get rid of wrongly detected objects:

A) Convert detected potential curbstones to a binary image. The grid increment must not be lower than the longitudinal laser point distance.

B) Perform a connectivity analysis for the curbstone pixels.

C) Outlier removal such as wrongly detected potential curbstones in binary image using their geometric properties. For such filtering we have considered first: length of the segments (connected cells of the binary image which show the potential position of curbstones) and secondly the parallelism of the segments. The vectorisation is applied by fitting proper spline on the potential curbstone positions. 


\section{RESULTS AND DISCUSSION}

The study area contains avenues and main streets with bikeways, park places and shallow curbstones. Such condition motivates us to examine road surface and boundary of the entire study area with our proposed methods. Two test areas (A and B) around the University of Applied Sciences in Stuttgart were selected and laser scanning test drives were carried out using FARO Focus3D X 330 laser scanner and Applanix POS LV 420 GNSS/INS system. Table 1 presents the characteristics of the laser scanner datasets.

\begin{tabular}{|c|c|c|c|}
\hline Dataset & $\begin{array}{c}\text { Length } \\
{[\mathrm{m}]}\end{array}$ & $\begin{array}{c}\text { Longitudinal } \\
\text { point distance }[\mathrm{m}]\end{array}$ & $\begin{array}{c}\text { Transverse point } \\
\text { distance }[\mathrm{m}]\end{array}$ \\
\hline $\mathrm{A}$ & 670 & 0.05 & 0.0025 \\
\hline $\mathrm{B}$ & 1100 & 0.04 & 0.004 \\
\hline
\end{tabular}

Table 1. Characteristics of the datasets

\subsection{Comparison of results of HDB and HB road surface detection}

Both HDB and HB variants together with the fourneighbourhood and two-neighbourhood region growing have their own pros and cons. In this study we have considered the results of HDB with four-neighbourhood and HB with twoneighbourhood. As advantage of four-neighbourhood region growing used in HDB variant, we noticed if there are cracks and potholes inside the road surface, the region growing shall not stop the process even if the height difference of these features is more than the threshold. The drawback of using this option is that if curbstone height from adjacent road surface is smaller than the predefined threshold, the region growing may count the sidewalks as road surface. Luckily using $2 \mathrm{~m}$ segment, less pedestrian area will be contaminated. Moreover because of the HDB functional which considers the absolute height difference, existence of objects above street floor such as car bumpers will terminate the process.

The superiority of HB variant with two-neighbourhood region growing is that due to the region growing along Y-axis, less pedestrian area will be detected as street floor (see Figure 5). Moreover street floor portion below car bumpers and bushes will be separated correctly from non-road surface area. Contrary to HDB variant with four-neighbourhood region growing, the existence of deep cracks, potholes and any other road surface depression terminates the process in the respective area. This is because of two neighbourhood region growing which is chosen in HD variant. Figure 5 shows the result of road surface detection using both conditions located in one of the most critical area. As presented in the Figure 5 (top), many parts of the sidewalks have been wrongly detected as street floor. On the other hand nearby both sides of zebra cross area, less sidewalks are wrongly detected as road surface in Figure 5 (down). This is because of two neighbourhood region growing. As nearby zebra cross we have shallow curbstones, it is more probable that the street floor extraction process fails.

\subsection{Comparison of the results with different thresholds}

The threshold in either of the variants (HDB and HD) plays an important role in the road surface detection process. Selecting small threshold value causes more time for region growing but it is worthwhile.

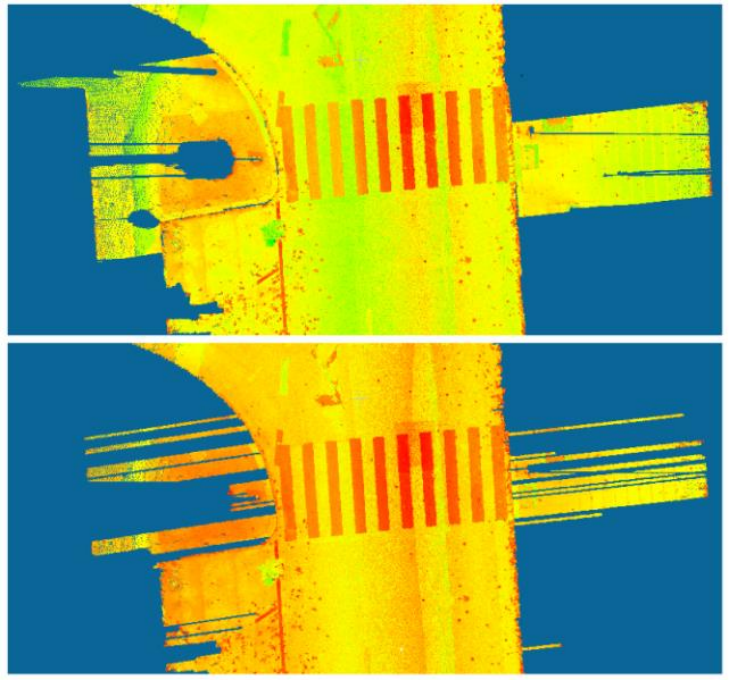

Figure 5. Comparison of HDB with four-neighbourhood region growing (top) and HD with two-neighbourhood region growing (down)

Figure 6 shows the result of road surface extraction using HD variant with two-neighbourhood region growing. The upper part of this figure looks neat compare to the lower sample. The reason is using smaller value for threshold. The area nearby the wheelchair sign is not properly separated and some sidewalks have been wrongly categorized as road surface. This is because of the shallow curbstones in that area. By reducing the voxel height (see Figure 6, top) we shall decrease the problem.

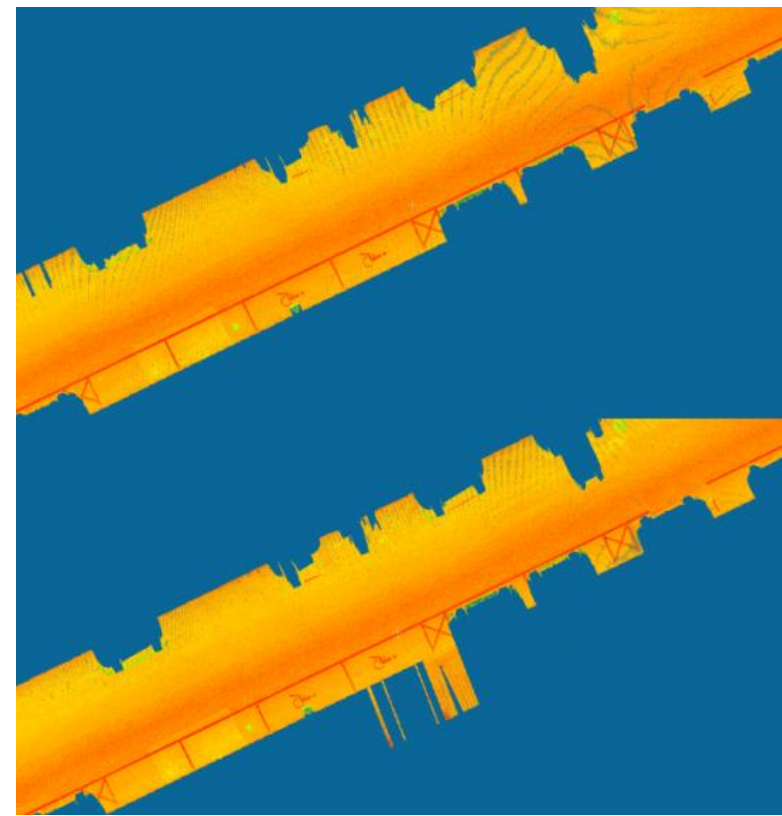

Figure 6. Comparison of road surface extraction using $2 \mathrm{~cm}$ (top) and $4 \mathrm{~cm}$ (down) voxel heights as threshold

\subsection{Vectorising the curb line position}

Generally it is important to apply post-processing to the result of potential curbstone detection. The post-processing aims to remove the wrongly selected regions such as car wheels (see Figure 7). Additionally due to the existence of obstacles like cars, the process of curbstone detection ends up having some gaps in the curbstone regions. As discussed, the obstacle 
removal is done by connectivity analysis. The gaps are filled and vectorisation is applied by fitting splines. The experimental investigations related to the curbstone detection show that apart from parking area and junctions, most of the vectorisations are done by fitting straight-lines to the potential curbstone positions.

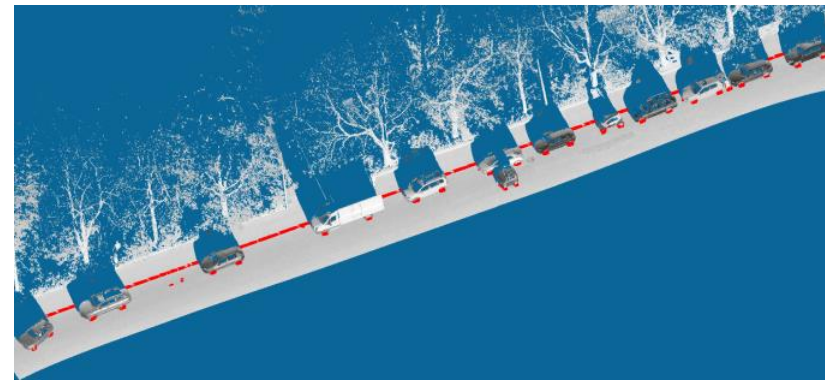

Figure 7. Extracted potential curbstone positions including outlier shown mostly as car wheels.

Figure 8 presents result of curbstone edge detection and vectorisation (green straight-lines) using $3 \mathrm{D}$ laser scanning point cloud. Figure 9 shows a situation in which the park place is located beside the zebra cross area. We have utilized the $4^{\text {th }}$ order spline to detect the curbstone edge.
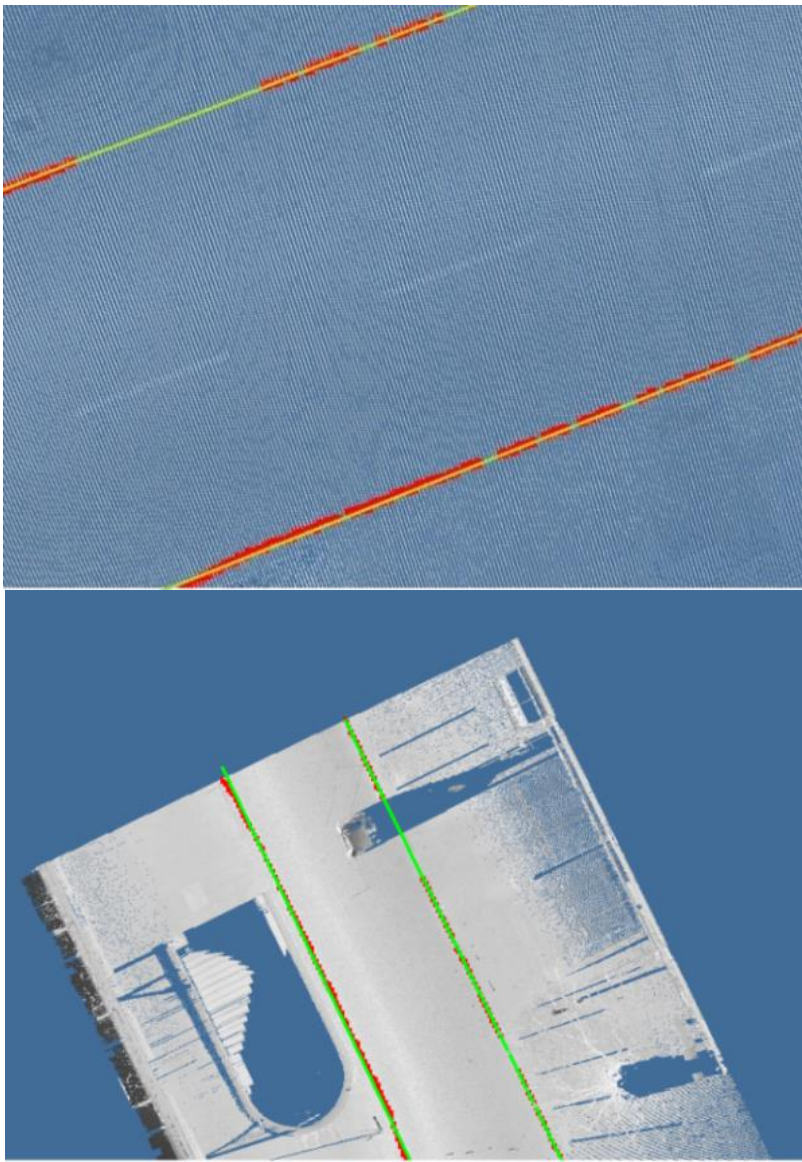

Figure 8. Result of curbstone edge detection and vectorisation (green straight-lines)

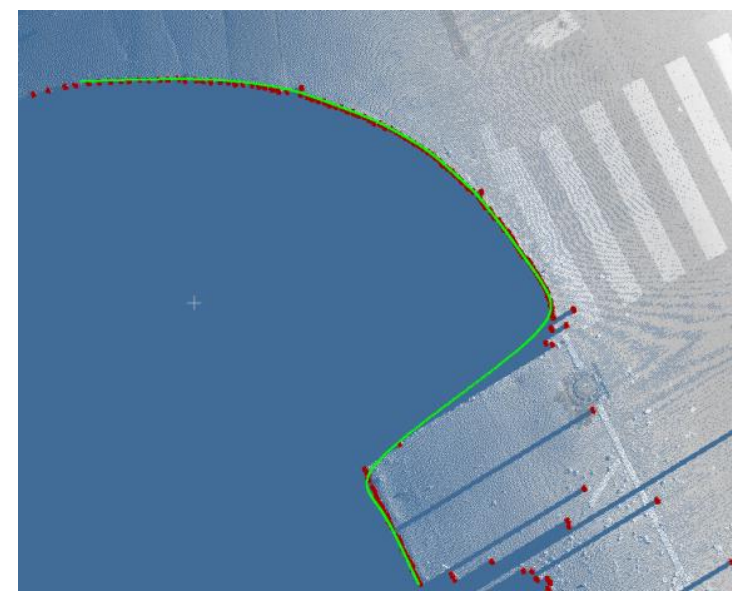

Figure 9. Result of curbstone edge detection and vectorisation using $4^{\text {th }}$ order spline

\subsection{Accuracy assessment}

We have compared results of road surface extraction using HDB and HB variants with ground truth data. Ground truth measurements are digitized manually from the laser scanner data. Percentage of both completeness and correctness are calculated using following formulae:

$$
\begin{aligned}
& \text { Correctness }=\frac{\text { correctly detected road surface }}{\text { correctly }+ \text { false detected road surface }} \\
& \text { Completeness }=\frac{\text { correctly detected road surface }}{\text { correctly }+ \text { not detecetd road surface }}
\end{aligned}
$$

Because of the existence of obstacles, some parts of the road surface are omitted. We have made use of dataset A (see Table 1) in such assessment. For the HD variant with twoneighbourhood region growing, the correctness value is $94.9 \%$ and completeness rate amounts to $93.8 \%$. Further, regarding HDB variant with four-neighbourhood region growing the correctness value is $92.4 \%$ and completeness rate amounts to 95.5\% (See Figure 10). As the dark blue area demonstrates the background; the area shown in red is the correctly-detected area. Bright blue regions show the wrongly detected and yellow areas present not-detected field. The omission error is more in HD because of the nature of two-neighbourhood region growing. Because of the existence of crack or extreme depression on the road surface, the process of two-neighbourhood region growing is terminated. Therefore in Figure 10 (down), some more yellow regions are obvious in the road surface. The commission error is more in HDB variant with four-neighbourhood region growing.

\section{SUMMARY AND FURTHER WORK}

In this study, two variants are introduced considering road surface and curbstone edge detection; HDB with fourneighbourhood and HB with two-neighbourhood. Boundaries of the detected road surfaces, as potential curbstone locations, are handled for further curb line detection. The results of the described procedures are used as input of the further research, i.e. road distress detection such as general unevenness, rut and pothole. The accuracy assessment of our results clarifies few manual refinement is necessary to remove wrongly extracted regions. The next step of our research in this area is to take the advantage of images which increases the possibility of automatic curbstone edge detection. 

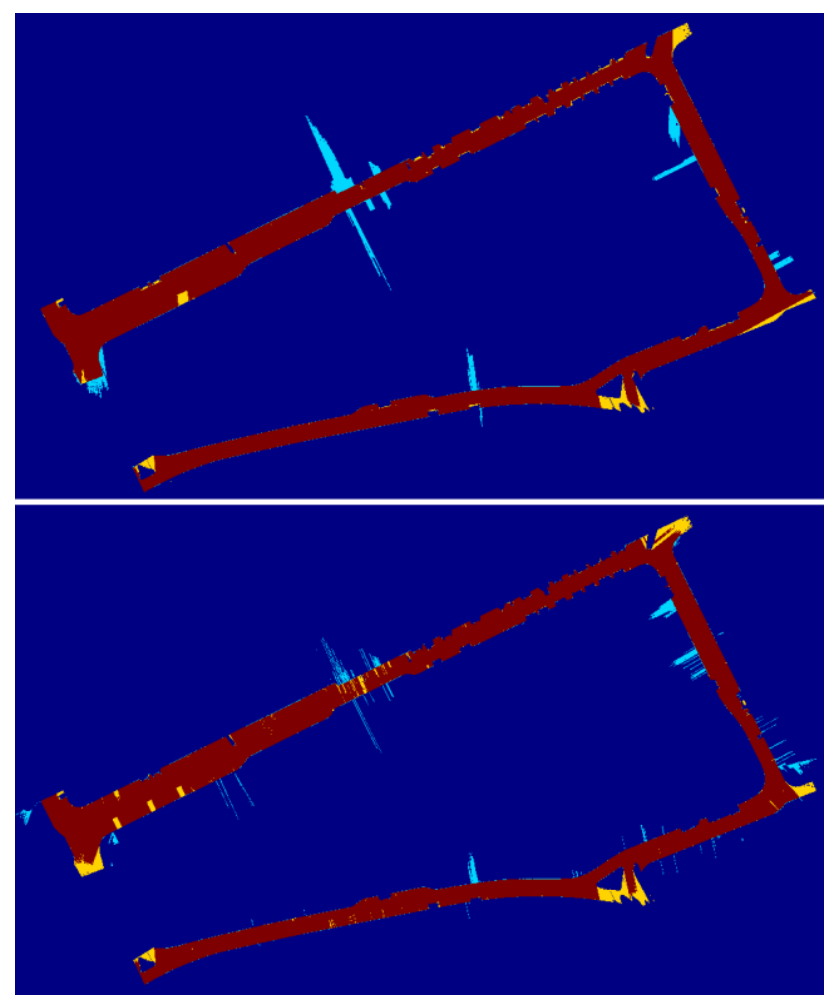

Figure 10. Accuracy assessment of automatic road surface extraction using HDB (top) and HB (down) variant

\section{ACKNOWLEDGEMENTS}

This work is funded by the German Federal Ministry of Education and Research within the project RoadInspect: Detection and assessment of road conditions with the help of the reflection spectroscopy - Development of a kinematic platform solution. Helpful discussions with Dr. Johannes Engels are highly acknowledged.

\section{REFERENCES}

El-Halawany, S., Moussa, A., Lichti, D. D., and El-Sheimy, N.: Detection of Road Curb from Mobile Terrestrial Laser Scanner Point Cloud, Int. Arch. Photogramm. Remote Sens. Spatial Inf. Sci., XXXVIII-5/W12, 109-114, doi:10.5194/isprsarchivesXXXVIII-5-W12-109-2011, 2011.

Gallo, O., Manduchi, R. and Rafii, A.: Robust curb and ramp detection for safe parking using the canesta ToF camera, Computer Vision and Pattern Recognition Workshops, CVPRW'08. Computer Society Conference. IEEE, 2008.

Michalke, T., Kastner, R., Fritsch, J. and Goerck, Ch.: A selfadaptive approach for curbstone/roadside detection based on human-like signal processing and multi-sensor fusion, Intelligent Vehicles Symposium (IV), IEEE, 2010.

Miraliakbari, A., Hahn, M., and Maas, H.-G.: Development of a Multi-Sensor System for Road Condition Mapping, Int. Arch. Photogramm. Remote Sens. Spatial Inf. Sci., XL-1, 265-272, doi:10.5194/isprsarchives-XL-1-265-2014, 2014.

Samadzadegan, F., Hahn, M. and Bigdeli, B.: Automatic Road Extraction from LIDAR Data based on Classifier Fusion, Urban Remote Sensing Joint Event, Joint. IEEE, 2009.
Siegemund, J., Pfeiffer, D., Franke, U. and Förstner, W.: Curb reconstruction using Conditional Random Fields, IEEE Intelligent Vehicles Symposium (IV), 2010.

Vosselman, G. and Liang, Z.: Detection of curbstones in airborne laser scanning data, Arch. Photogramm. Remote Sens. Laser scanning 2009, ISPRS, Vol. XXXVIII, Part 3/W8, 2009.

Wikipedia, Curb, http://en.wikipedia.org/wiki/Curb\#cite_note-6 (Access 15.04.2014) 